\title{
Oblique Electroweak Corrections From Heavy Scalar Fields
}

\author{
Ling-Fong $\mathrm{Li}$ \\ Physics Department \\ Carnegie Mellon University \\ Pittsburgh, PA 15213
}

\begin{abstract}
The contributions of heavy scalar fields to the oblique electroweak correction parameters $S$ and $T$ are discussed with an emphasis of the decoupling properties of these contributions. It turns that in the decoupling limit, we also get the custodial symmetry.
\end{abstract}

\section{DISCLAIMER}

This report was prepared as an account of work sponsored by an agency of the United States Government. Neither the United States Gnvernment nor any agency thereof, nor any of their emplojees, makes any warranty, express or implied, or assumes any legal liability or responsibility for the accuracy, completeness, or usefulness of any information, apparatus, product, or process disclosed, or represents that its use would not infringe privately owned rights. Reference herein to any specific commercial product, process, or service by trade name, trademark, manufacturer, or otherwise does not necessarily constitute or imply its endorsement, recommendation, or favoring by the United States Government or any agency thereof. The views and opinions of authors expressed herein do not necessarily state or reflect those of the United States Government or any agency thereof. 


\section{Introduction}

The study of precision measurements in the electroweak interactions can test the detailed structure of the Standard Model(SM) and look for new physics. In particular, over the years, the higher order radiative corrections in electromagnetic interaction has played an important role in establishing the QED as the fundamental theory. The recent analysis on the oblique electroweak corrections ${ }^{1,2,3}$ can provides useful information about the possible extensions of the Standard Model of electroweak interactions.

One of the interesting features of these oblique corrections is that they are quite sensitive to the heavy particles in many of the extensions of the Standard Model. These sensitive dependence on the high masses, which are results of violation of the decoupling theorem, ${ }^{4}$ might provide a window to study: physics at the high mass scales. On the other hand, the violation of decoupling is somewhat disturbing as it implies that physics at a given mass scale can not be studied in isolation. One possible way to avoid the situation is to have those particles which violate the decoupling to be not too heavy while very heavy particles should be implemented in such a way that the decoupling is salisfied. 5

In the standard model, the fermions get their masses from the Yukawa couplings to the Higgs scalars. Thus the masses heavier than the Higgs vacuuam expectation values (VEV) will require large Yukawa couplings, which are the source of the decoupling violations. Fermions much heavier than the electroweak scale most likely will get their masses from either bare mass term or some VEV's much larger than the electroweak scale. In these cases the physics at the electroweak scale will not be sensitive to the very heavy particles.

The contribution from the heavy fermions to the oblique corrections has been studied quite extensively in the literature. In this paper we will discuss some general feature of the scalar contributions. In particular, the mechanism for the decoupling the heavy scalar particles will be studied. For the purpose of comparison we summarize the some of the results for the fermion contributions with some remarks.

It is well known that the $\mathrm{T}$ parameter ${ }^{\prime}$ in the oblique corrections measures the violation of the custodial $S U(2)$ symmetry and is positive for a standard left-handed lieavy doublet.' On the other hand the present experimental data seems to favor a negative value for $T^{\prime}{ }^{-}$It was pointed out that for multiplets involving Majorana particles the parameter $\mathrm{T}$ can take negative values. ${ }^{8}$ Actually, it is not hard to see that 
the negative contribution comes mainly from the interierence between the left-handed and the right-handed $\mathrm{SU}(2)$ gauge couplings in the self energy graphs of the gauge bosons. 9,11 Thus the positiveness of the T parameter holds only for special cases of the pure left-handed (or right-handed) gauge couplings. Therefore, the $T$ parameter can take negative values if both the left-handed and right-handed components transform non-trivially under the $S U(2)_{L}$ gauge group.

It is interesting to note that for the case of left-handed doublet, the $T$ parameter depends on the heavy fermion masses quadratically and this violates the decoupling theorem. This is not surprising because in this case the large fermion mass comes from a large Yukawa coupling which invalidates the decoupling. One way to recover the decoupling is to introduce the heavy fermions in the form of vector-like multiplets so that the heavy mass can come from a large bare mass without large coupling. In these cases the $\mathrm{T}$ parameter will vanish in the heavy mass limit due to some complicated cancellation." One important feature in the calculation is the fact that the mixing betweer the light and heavy fermions is inversely proportional to heavy mass.

The $\mathrm{S}$ parameter in the oblique correction which is proportional to the combination $\pi_{31}^{\prime} \cdot(0)$ of the gauge boson self energies, is given for the usual left-handed doublet as

$$
S=\frac{1}{6 \pi}\left(-Y \ln \frac{m_{u}^{2}}{m_{d}^{2}}+1\right)
$$

where $\mathrm{Y}$ is the weak hypercharge and $m_{u}, m_{d}$ are the masses of up and down components of the doublet. Thus the sign of $S$ is positive for small mass splitting in the doublet and could be negative for large mass splitting. It turns out that $S$ vanishes for the left-right symmetric multiplets in the limit of degenerate masses. ${ }^{9}$ Thus in some sense, the $\mathrm{S}$ parameter measures the asymmetry between the left-handed and the right-handed fields. Hence one can make the $S$ parameter small by having an approximate left-right symmetry, i.e. vector-like multiplets with respect to the $\mathrm{SU}(2)_{L}$ gauge group.

From Eq.(1) we see that for the case of the usual doublet the $S$ parameter depends on the heavy masses only logarithmically. This implies that if we add a heavy vectorlike $\mathrm{SU}(2)$ singlet, the $\mathrm{S}$ parameter will vanish in the limit of large mass because the heavy fermion can couple to the $\mathrm{SU}(2)$ gauge bosons only through mixing which is inversely proportional to the heavy mass. The decoupling should be valid for the more general cases as long as the large mass does not come from large couplings. 
The scalar contribution to the $\mathrm{T}$ parameter has been calculated many years ago by Toussiant" in the two doublet extension of the Stundard Model . The result is that it can have either sign and it grows as we increase the Higgs mass. This violation of decoupling is due tu the fact that in this case the large Higgs mass requires a large scaiar self coupling. The sign of the $S$ parameter has been studied recently in the limit of the custodial SU(2) symmetry. ${ }^{12}$

In this paper we will study the oblique parameters $S$ and $T$ for the cases where the large scalar mass does not come from large coupling so that the decoupling theorem holds. It is expected that the dependence on the scalar masses of these parameters will be strongly influenced by the decoupling.

\section{Heavy Scalar Particles}

In this section we will specify the properties of the scalar particles who:e contribution to the oblique parameters, $S, T$, will be studied in the next section. II we will be interested in the validity of the decoupling of the scalar particles in the oblique corrections. Specifically, we are interested in the limit masses of the scalar particles are much greater than the vacuum expectation value(VEV), $v$, that breaks the $\mathrm{SU}(2) \times \mathrm{U}(1)$ symmetry. Since VEV of any scalar in the nontrivial representation of $\mathrm{SU}(2)$ group will contribute to the gauge boson masses, which are held fixed, the heavy scalar particles can be realized without large couplings only for those which do not develop VEV. This can be achieved by changing the sign of the quadratic terms in the Higgs potentials. For simplicity, we will introduce an $S U(2)$ doublets of scalar particles in addition to the standard model doublet. With appropriate discrete symmetry, the Higgs potentials can be written as,

$$
\begin{gathered}
V=-\mu^{2} H^{+} H+\frac{\lambda_{1}}{2}\left(H^{+} H\right)^{2}+m^{2} \phi^{+} \phi+\frac{\lambda_{2}}{2}\left(\phi^{+} \phi\right)^{2} \\
+\lambda_{3}\left(H^{+} H\right)\left(\phi^{+} \phi\right)+\lambda_{4}\left(H^{+} \phi\right)\left(\phi^{+} H\right)+\lambda_{5}\left[\left(H^{+} \phi\right)\left(H^{+} \phi\right)+\text { h.c. }\right]
\end{gathered}
$$

where $H$ is the standard doublet and

$$
\phi=\left(\begin{array}{l}
\phi^{\circ} \\
\phi^{-}
\end{array}\right)
$$

is the new doublet. Note that the quadratic term in $\phi$ has the opposite sign from that of $\mathrm{H}$, so that it is possible to have a solution for the minimum of the Higgs potential in which the scalar field $\phi$ do not develop VEV. Befare the spontaneous symmetry breaking (SBS), both particles in the doublet $\phi$ have the same mass $m$, and SBS will 
split the masses, denoted by $m_{1}, m_{2}$, through the coupling between $\mathrm{H}$ and $\phi$. Thus we have

$$
\left|m_{1}^{2}-m_{2}^{2}\right| \propto v^{2} \ll m^{2}
$$

i.e. in the heavy mass limit two scalar particles become degenerate again. This is a general feature of the heavy particles, if their heavy masses do not come from large couplings. Note that in this simple case there is no mixing between $\mathrm{H}$ and $\phi$ multiplets which simplifies the calculation.

It is interesting to note that in the heavy mass limit specified by Eq.(4), we also have the custodial SU(2) symmetry for the scalar doublet. As we shall see later that this will have the consequence that the decoupling of the heavy particles in the oblique parameters will imply that these parameters will also vanish in the limit of exact $S U(2)$ symmetry. This feature should be true for all multiplets, fermions or bosons, as long as heavy mass limit does not involve large couplings. For example, the S parameter given :- Eq.(1), which satisfiies both the decoupling and the custodial symmetry in the limit given in Eq.(4).

\section{Scalar contribution to $S$, and $T$ parameters}

The 1-loop contributions to the gauge boson self energies come from diagrams given in Fig. 1 and Fig. 2. These two types of diagrams gives rise to the following two integrals,

$$
\begin{gathered}
I_{1}^{\mu \nu}(m)=\frac{i g^{2}}{2} g^{\mu \nu} \int \frac{d^{4} k}{(2 \pi)^{4}} \frac{1}{k^{2}-m^{2}} \\
I_{2}^{\mu \nu}\left(m_{1}, m_{2}, q\right)=\left(\frac{-i g^{2}}{4}\right) \int \frac{d^{\prime \prime} k}{(2 \pi)^{4}} \frac{(2 k+q)^{\mu}(2 k+q)^{\nu}}{\left(k^{2}-m_{1}^{2}\right)\left[(k+q)^{2}-m_{2}^{2}\right]}
\end{gathered}
$$

For the calculation of the $\mathrm{T}$ parameter we can set $q=0$. In dimensional regulation, these are given by

$$
\begin{gathered}
I_{1}^{\mu \nu}(m)=g^{2} \frac{1}{2} g^{\mu \nu} \frac{1}{(4 \pi)^{2}} m^{2}\left[\xi+\left(1-\ln \frac{m^{2}}{\mu^{2}}\right)\right] \\
I_{2}^{\prime \prime \nu}\left(m_{1}, m_{2}, 0\right)=-g^{2} \frac{1}{4} g^{\prime \prime \prime \prime} \frac{1}{(4 \pi)^{2}}\left\{\left(m_{1}^{2}+m_{2}^{2}\right)\left(\xi+\ln \mu^{2}\right)+\left(m_{1}^{2}+m_{2}^{2}\right)\right. \\
\left.-2 \int_{11}^{1} d \alpha\left[a m_{1}^{2}+(1-\alpha) m_{2}^{2}\right] \ln \left[\alpha m_{1}^{2}+(1-\alpha) m_{2}^{2}\right]\right\}
\end{gathered}
$$


where $\mu$ is the arbitrary mass parameter in the dimensional regulation and $\xi$ is the combination

$$
\xi=\frac{1}{\epsilon}-\gamma+\ln 4 \pi
$$

with $\gamma$ the Euler's constant, and $\epsilon=(4-n) / 2$. Using these two functions we can calculate the scalar contrihutions to the oblique parameter $\mathrm{T}$ given by

$$
\alpha T=\Delta \rho=\frac{e^{2}}{\sin ^{2} \theta_{1 \prime} M_{w \prime}^{2}}\left[\pi_{11}(0)-\pi_{33}(0)\right]
$$

The indices 1 and 3 refer to $\mathrm{SU}(2)_{L}$ components and $g^{2} \pi_{i j}\left(q^{2}\right)$ is the self energy of $\mathrm{SU}(2)$ gauge bosons. After some algebra, the result is given by

$$
\begin{gathered}
\pi_{11}(0)=\frac{1}{32 \pi^{2}}\left[\frac{m_{1}^{2} m_{2}^{2}}{\left(m_{2}^{2}-m_{1}^{2}\right)} \ln \frac{m_{1}^{2}}{m_{2}^{2}}+\frac{1}{2}\left(m_{1}^{2}+m_{2}^{2}\right)\right] \\
\pi_{: 33}(0)=0
\end{gathered}
$$

Eq.(12) follows from the fact that for the case $m_{1}=m_{2}=m, I_{1}^{\mu \nu}$ and $I_{2}^{\mu \nu}$ cancel each other. This simply corresponds to the fact that for equal masses, $\pi_{3: 3}$ reduces to the photon self energy which vanishes at $q^{2}=0$. The $\mathrm{T}$ parameter is then given by

$$
\alpha T=\frac{e^{2}}{\sin ^{2} \theta_{w} M_{w 1}^{2}} \times \frac{1}{32 \pi^{2}}\left[\frac{m_{1}^{2} m_{2}^{2}}{\left(m_{2}^{2}-m_{1}^{2}\right)} \ln \frac{m_{1}^{2}}{m_{2}^{2}}+\frac{1}{2}\left(m_{1}^{2}+m_{2}^{2}\right)\right]
$$

It is interesting to note that this result is exactly the same as the fermion contribution to $\mathrm{T}$ parameter for a single $\mathrm{SU}(2)$ left-handed doublet and is positive except for $m_{1}=m_{2}$. To see how the decoupling takes place for the scalar contribution, we let $m_{1}, m_{2}$ be large but with $\left|m_{1}^{2}-m_{2}^{2}\right| \ll m_{1}^{2}+m_{2}^{2}$, as explained in last section. In this limit we get

$$
\alpha T=\frac{e^{2}}{\sin ^{2} \theta_{u} \cdot M_{1 \prime}^{2}} \frac{1}{8 \pi^{2}} \times \frac{1}{3} \frac{\left(\Delta m^{2}\right)^{2}}{\left(m_{1}^{2}+m_{2}^{2}\right)}
$$

where $\Delta m^{2}=m_{1}^{2}-m_{2}^{2}$. Clearly, the decoupling is valid for this case. Note that even though the mass dependence here is the same as the fermion case, the decoupling features are completely different. For the left-handed doublet, the masses $m_{1}, m_{2}$ rome from independent Yukawa couplings and the decoupling fails if either mass hecnme very la.rge. Note that $\mathrm{T}$ in Eq.(14) also vanishes in the custodial $S U(2)$ limit as it should.

In the more general cases, there might be mixing between the light and heavy particles. However, this will not change the decoupling feature. This can be seen 
as follows. For the graphs involving only the heavy particles, the analysis is the same as before and those containing only light particles are irrelevant.For the graphs containing one light and one heavy particles, we can take the limit, $m_{1}$ large and $m_{2}$ fixed in Eq.(13) to get

$$
T \propto m_{1}^{2}
$$

which seems to grow in the large mass limit. However, it is not hard to see that the mixings between the light and heavy particles are suppressed by $\left(m_{2}^{2} / m_{1}^{2}\right)$, which comes in twice in the self energy graphs. These factors will cause the parameter $T$ to go to zero in the heavy mass limit and decoupling is recovered. Note that in the case with mixing $\pi_{33}(0)$ is no longer zero because the gauge coupling of $W_{3}^{\mu}$ is no longer diagonal in the mass eigenstates. But it is easy to see that the mass dependence of $\pi_{: 33}(0)$ is similar to that of $\pi_{11}(0)$ and the same conclusion holds.

For the calculation of the $S$ parameter we write Eq. (6) as

$$
I_{2}^{\mu \nu}\left(m_{1}, m_{2}, q\right)=g^{2} g^{\prime \nu} \pi_{2}\left(m_{1}, m_{2}, q^{2}\right)+\ldots
$$

From this we can calculate the first derivative with respect to $q^{2}$,

$$
\begin{gathered}
\left.\pi_{2}^{\prime}\left(m_{1}, m_{2}\right) \equiv \frac{d \pi_{2}\left(m_{1}, m_{2}, q^{2}\right)}{d q^{2}}\right|_{q^{2}=0} \\
=\frac{1}{(4 \pi)^{2}} \cdot \frac{1}{4}\left\{-\frac{1}{3}\left(\xi+\ln \mu^{2}\right)+2 \int_{0}^{1} d \alpha \alpha(1-\alpha) \ln \left[\alpha m_{1}^{2}+(1-\alpha) m_{2}^{2}\right]\right\}
\end{gathered}
$$

The $S$ parameter is then given by

$$
S=-\left.8 \pi \frac{d}{d q^{2}} \pi_{3 Y}\left(q^{2}\right)\right|_{q^{2}=0}=\frac{1}{24 \pi} \ln \left(\frac{m_{1}^{2}}{m_{2}^{2}}\right)
$$

where $m_{1}$ and $m_{2}$ are masses of $\phi^{\circ}$ and $\phi^{-}$, respectively. It is clear from this that in the large mass limit defined by Eq.(4), $S$ goes to zero and the decoupling is valid. The new feature here is that $S$ vanishes also in the limit of exact custodial $S U(2)$ symmetry as a consequence of decoupling as explained in the previous section.

\section{Discussion}

We have studied the oblique corrections coming form an $S U(2)$ doublet of scalars which does not have VEV's so that their masses can be much heavier than the electroweak scale. As a consequence, in the heavy mass limit the mass difference in the multiplet has to be much smaller than the masses themselves. Thus in the heavy mass limit, we have the custodial SU(2) symmetry. This should be true for all multiplets for which the decoupling theorem is valid. Thus the decoupling of the heavy 
particles also implies that their contribution to the oblique corrections vanish in the custodial SU(2) symmetry limit. The decoupling properties of the parameters $S, T$ are demonstrated explicitly. It is interesting to note that the scalar doublet contribution to the parameter $T$ is exactly the same as the left-handed doublet contribution and is positive. The $S$ parameter from the scalar can have either sign. But the new features is that it vanishes in the degenerate mass limit.

This work was supported in part by the United States Department of Energy, under the contract DE-FG02-91ER-40682. 


\section{References}

1. M.E. Peskin and T. Takeuchi, Phys. Rev. Lett. $\underline{65}, 964$ (1990).

2. M. Golden and L. Randall, Nucl. Phys. B361, 3 (1991).

3. G. Altarelli and R. Barbieri, Phys Lett. B253, 161 (1991); B. Grinstein and M. Wise, Phys Lett. B265, 326 (1991); M. E. Peskin and T. Takeuchi, Phys. Rev. D46, 381 (1992).

4. T. Applequist and J. Carazzone, Phys. Rev. D11, 2856 (1975).

5. See for example, T.P. Cheng, and L.F. Li, Phys. Rev. D44, 1502 (1991).

6. M. Veltman, Nucl. Phys. B123, 89 (1977).

7. D. C. Kennedy and P. Langacker, Phys. Rev. Lett. $\underline{65} .2967$ (1990); 66, 395(E) (1991); W. J. Marciano and J. Rosner, Phys. Rev. Lett. $\underline{65}, 2963$ (1990).

8. S. Bertolini and A. Sirlin, Phys. Lett. $\underline{\text { B257, }} 179$ (1991).

9. E. Gates and J. Terning, Phys. Rev. Lett. $\underline{67}, 1840$ (1991).

10. E. Ma. and P. Roy, Phys. Rev. Lett. $\underline{68}, 2879$ (1992).

11. D. Toussiant, Phys. Rev. D18, 1626 (1978).

12. M. Dugan and L. Randall, Phys. Lett. B264, 154 (1991).

\section{Figure Caption}

Fig.1 the gauge boson self energy graph coming from the tri-linear coupling.

Fig.2 the gauge boson self energy graph coming from the quartic coupling. 


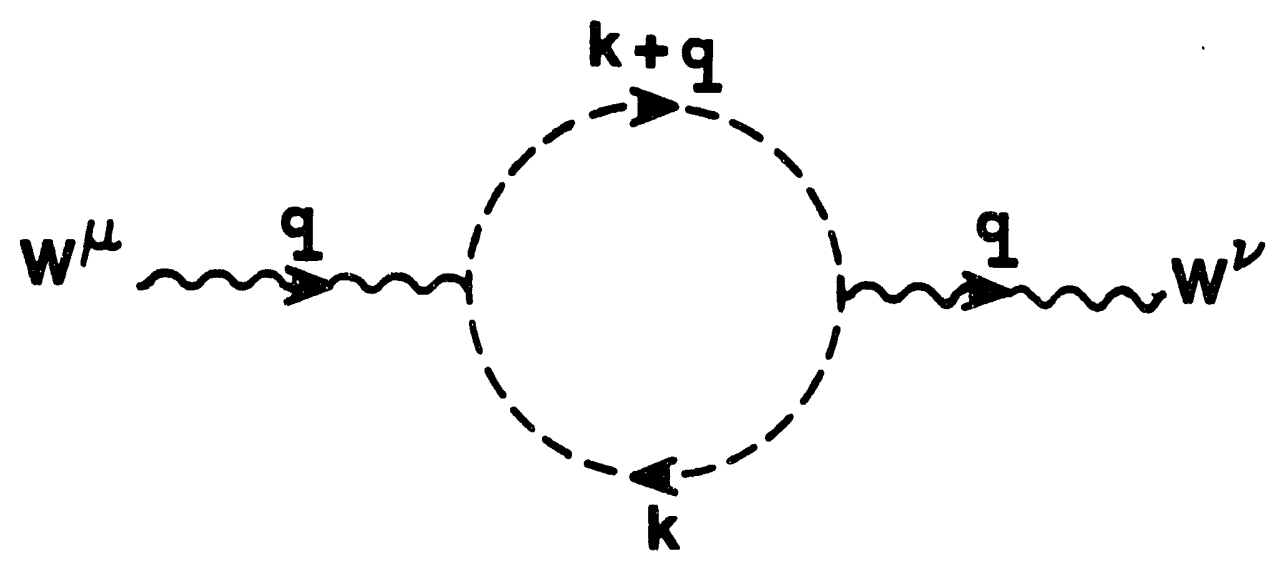

Fig. 1

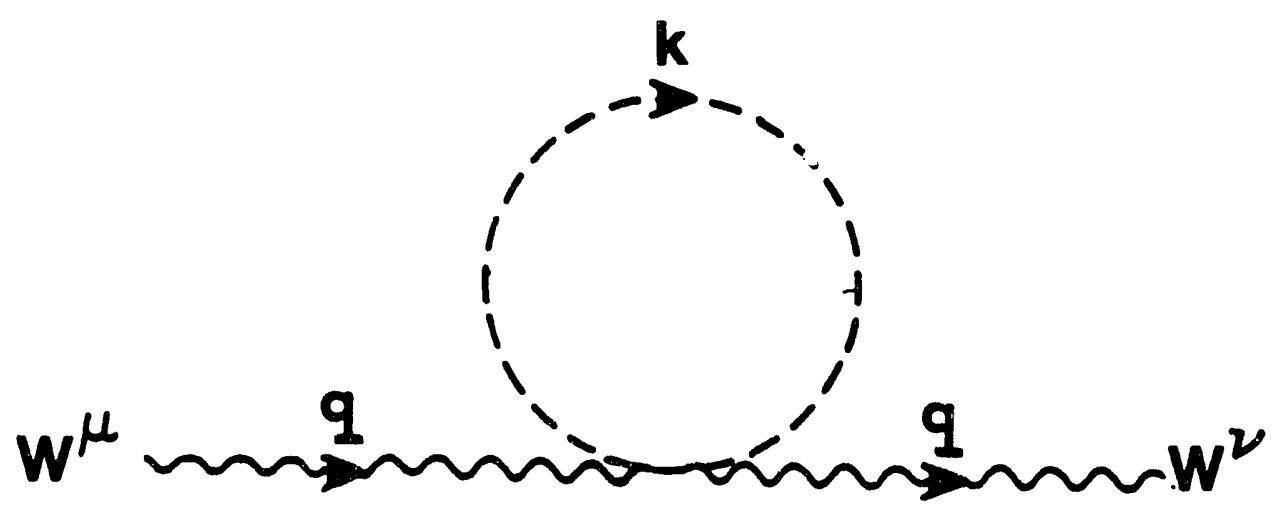

Fig. 2 

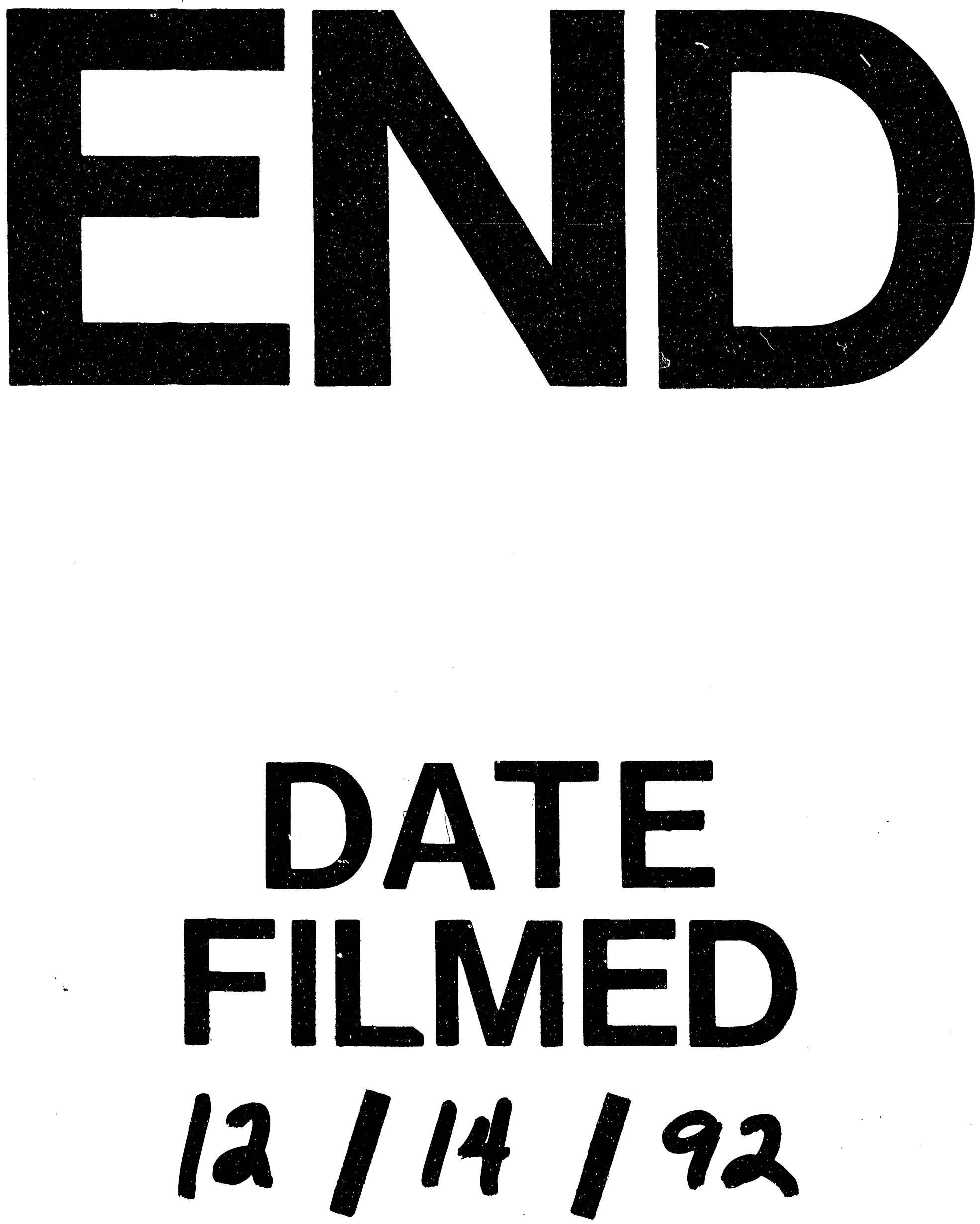
